\title{
A New 5-Point Ternary Interpolating Subdivision Scheme and Its Differentiability
}

\author{
Ghulam Mustafa, Jayyada Irum, and Mehwish Bari \\ Department of Mathematics, The Islamia University of Bahawalpur, Bahawalpur 63100, Pakistan \\ Correspondence should be addressed to Ghulam Mustafa, ghulam.mustafa@iub.edu.pk
}

Received 25 July 2012; Accepted 18 September 2012

Academic Editors: L. Hajdu, L. Pan, and Q.-W. Wang

Copyright ( $) 2012$ Ghulam Mustafa et al. This is an open access article distributed under the Creative Commons Attribution License, which permits unrestricted use, distribution, and reproduction in any medium, provided the original work is properly cited.

A new 5-point ternary interpolating scheme with a shape parameter is introduced. The resulting curve is $C^{2}$ for a certain range of parameters. The differentiable properties of the proposed scheme to extend its application in the generation of smooth curves are explored. Application of the proposed scheme is given to show its visual smoothness. The scheme is also extended to a 5-point tensor product ternary interpolating scheme, and its numerical examples are also included.

\section{Introduction}

Geometric modeling plays a significant role to cover up the gap between computer and industry. It has a pivotal importance in the fields of aircraft manufacturing, automobile industry, and general product design.

One of the most important tools of computer aided geometric design is "Subdivision." Subdivision is a well flourished field. It is a process of taking unrefined shape and to polish it up to produce another shape that is more visually tempting. Due to the comprehensibility and simplicity of this method, it is used in the fields of 3D geometrical measurement, computer graphics, computer animation, and computer aided geometric design.

In 1986, Dubuc [1] presented a interpolation through an iterative scheme. Dyn et al. [2] introduced a 4-point interpolating subdivision scheme for curve design. Later on, Deslauriers and Dubuc [3] introduced a symmetric iterative interpolation process. Weissman [4] also offered a 6-point interpolating scheme in 1990. In 2002, Hassan et al. [5, 6] gave ternary three-point and 4-point interpolatory schemes. Further analysis of ternary three-point univariate scheme was given in technical report by Hassan and Dodgson [7] in 2004. Dyn [8] has given the analysis of the convergence and smoothness of interpolating and approximating schemes by Laurent's polynomial method.
In 2007, Beccari et al. [9] presented an interpolating 4point ternary nonstationary scheme with tension control. They also offered a nonstationary uniform tension controlled interpolating 4-point scheme reproducing conics [10] in 2007. Ko [11] in his Ph.D. thesis presented a detailed study on subdivision scheme. Zheng et al. [12] presented the method to find the differentiability of a four-point ternary scheme. Lian [13] extended 3-point and 5-point interpolating schemes into $a$-ary subdivision scheme for curve design. Conti et al. [14] derived symmetric subdivision masks of the Hurwitz type to the interpolating scheme masks. In this paper, we present a new 5-point ternary interpolating subdivision scheme with one parameter.

\section{Preliminaries}

Let $f_{i}^{k} \in \mathbb{R}^{N}, i \in \mathbb{Z}$, denote a sequence of points in $\mathbb{R}^{N}$, $N \geqslant 2$, where $k$ is a nonnegative integer, then a univariate ternary subdivision scheme $S$ which maps coarse points $f_{i}^{k}$ to refined points $f_{i}^{k+1}$ is defined by

$$
\begin{aligned}
f_{3 i}^{k+1} & =\sum_{j=0}^{m} a_{3 j} f_{i+j}^{k}, \\
f_{3 i+1}^{k+1} & =\sum_{j=0}^{m} a_{3 j+1} f_{i+j}^{k},
\end{aligned}
$$




$$
f_{3 i+2}^{k+1}=\sum_{j=0}^{m} a_{3 j+2} f_{i+j}^{k},
$$

where $m>0$ and the set $a=\left\{a_{i} \mid i \in \mathbb{Z}\right\}$ of coefficients is called mask of the scheme. The $z$-transform of the mask $a$ of subdivision scheme $S$ can be given as

$$
a(z)=\sum_{i \in \mathbb{Z}} a_{i} z^{i},
$$

which is called the symbol or the Laurent polynomial of the scheme. The Laurent polynomial of a convergent subdivision scheme satisfies

$$
a\left(e^{2 i \pi / 3}\right)=a\left(e^{4 i \pi / 3}\right)=0, \quad a(1)=3 .
$$

The existence of associated subdivision scheme $S_{n}$ to $S$ for the divided differences of the original control polygon and of related Laurent polynomial $a_{n}(z)$ is assured by the following condition:

$$
\begin{gathered}
a_{n}(z)=\left(\frac{3 z^{2}}{z^{2}+z+1}\right) a_{n-1}, \\
(z)=\left(\frac{3 z^{2}}{z^{2}+z+1}\right)^{n} a(z), \quad n \geqslant 1, \\
\left\|\left(\frac{1}{3} S_{n}\right)^{L}\right\|_{\infty}=\max \left\{\sum_{j \in \mathbb{Z}}\left|b_{i+3^{L} j}^{[n, L]}\right|: i=0,1, \ldots, 3^{L}-1\right\},
\end{gathered}
$$

where

$$
b^{[n, L]}(z)=\frac{1}{3^{L}} \prod_{j=0}^{L-1} a_{n}\left(z^{3^{j}}\right) .
$$

By Hassan and Dodgson [6], $C^{n}$ continuity of the scheme $S$ requires that $a_{n}(z)$ satisfy $(4)$ and $\left\|\left((1 / 3) S_{n+1}\right)^{L}\right\|_{\infty}<1$, for the first integer value of $L>0$.

\section{A 5-Point Ternary Interpolating Scheme}

In this section, we construct a 5-point ternary interpolating subdivision scheme and give its analysis.

3.1. Construction of the Scheme. Consider the following three recursive relations which refine given the $k$ th level polygon $f^{k}=\left\{f_{i}^{k}\right\}_{i \in \mathbb{Z}}$ to $(k+1)$ th level polygon $f^{k+1}=\left\{f_{i}^{k+1}\right\}_{i \in \mathbb{Z}}$

$$
\begin{gathered}
f_{3 i-1}^{k+1}=a_{0} f_{i-2}^{k}+a_{1} f_{i-1}^{k}+a_{2} f_{i}^{k}+a_{3} f_{i+1}^{k}+a_{4} f_{i+2}^{k}, \\
f_{3 i}^{k+1}=f_{i}^{k}, \\
f_{3 i+1}^{k+1}=a_{4} f_{i-2}^{k}+a_{3} f_{i-1}^{k}+a_{2} f_{i}^{k}+a_{1} f_{i+1}^{k}+a_{0} f_{i+2}^{k} .
\end{gathered}
$$

We have the following sequence from the above recurrence relation:

$$
\begin{array}{r}
a=\left\{\ldots, 0, a_{4}, 0, a_{0}, a_{3}, 0, a_{1}, a_{2},\right. \\
\left.1, a_{2}, a_{1}, 0, a_{3}, a_{0}, 0, a_{4}, 0, \ldots\right\} .
\end{array}
$$

The Laurent polynomial of this sequence is

$$
\begin{aligned}
a(z)= & a_{4} z^{7}+a_{0} z^{5}+a_{3} z^{4}+a_{1} z^{2}+a_{2} z+1 \\
& +a_{2} z^{-1}+a_{1} z^{-2}+a_{3} z^{-4}+a_{0} z^{-5}+a_{4} z^{-7} .
\end{aligned}
$$

Let $\left\{S_{i}: i=0,1,2, \ldots\right\}$ be the divided difference subdivision schemes of $S$ corresponding to the Laurent polynomial $a_{i}(z)$, then by Hassan and Dodgson [6], the necessary condition for $C^{i}$ continuity of scheme $S$ is that $a_{i}(z)$ satisfy (3), which gives for $i=0,1,2,3$ and by (4)

$$
\begin{aligned}
& a_{0}+a_{1}+a_{2}+a_{3}+a_{4}=1, \\
& 6 a_{0}+3 a_{1}-3 a_{3}-6 a_{4}=1, \\
& 36 a_{0}+9 a_{1}+9 a_{3}+36 a_{4}=1, \\
& -9 a_{0}-9 a_{3}-27 a_{4}=1, \\
& -54 a_{0}+27 a_{3}+162 a_{4}=1, \\
& 162 a_{0}-162 a_{4}=-8, \\
& a_{1}(z)=3 a_{4} z^{7}-3 a_{4} z^{6}+3 a_{0} z^{5}+3\left(a_{3}+a_{4}-a_{0}\right) z^{4} \\
& -3\left(a_{3}+a_{4}\right) z^{3}+3\left(a_{0}+a_{1}\right) z^{2} \\
& +3\left(1-2 a_{0}-2 a_{1}\right) z+3\left(a_{3}+a_{4}-a_{0}\right) z^{-2} \\
& +3\left(a_{0}+a_{1}\right)-3\left(a_{3}+a_{4}\right) z^{-1} \\
& +3 a_{0} z^{-3}-3 a_{4} z^{-4}+3 a_{4} z^{-5}, \\
& a_{2}(z)=9 a_{4} z^{7}-18 a_{4} z^{6}+9\left(a_{0}+a_{4}\right) z^{5} \\
& +9\left(a_{3}+2 a_{4}-2 a_{0}\right) z^{4}+9\left(a_{0}-2 a_{3}-4 a_{4}\right) z^{3} \\
& +9\left(2 a_{0}+a_{1}+a_{3}+2 a_{4}\right) z^{2}+9\left(a_{0}-2 a_{3}-4 a_{4}\right) z \\
& +9\left(a_{3}+2 a_{4}-2 a_{0}\right) \\
& +9\left(a_{0}+a_{4}\right) z^{-1}-18 a_{4} z^{-2}+9 a_{4} z^{-3}, \\
& a_{3}(z)=27 a_{4} z^{7}-81 a_{4} z^{6}+\left(27 a_{0}+81 a_{4}\right) z^{5} \\
& +\left(-81 a_{0}+27 a_{3}+54 a_{4}\right) z^{4} \\
& +\left(81 a_{0}-81 a_{3}-243 a_{4}\right) z^{3} \\
& +\left(-81 a_{0}+27 a_{3}+54 a_{4}\right) z^{2} \\
& +\left(27 a_{0}+81 a_{4}\right) z-81 a_{4}+27 a_{4} z^{-1}, \\
& a_{4}(z)=27 a_{4} z^{7}-108 a_{4} z^{6} \\
& +\left(27 a_{0}+162 a_{4}\right) z^{5}+\left(-108 a_{0}+27 a_{3}\right) z^{4} \\
& +\left(27 a_{0}+162 a_{4}\right) z^{3}-108 a_{4} z^{2}+27 a_{4} z .
\end{aligned}
$$

Solving the system of liner equation (9) by taking $a_{4}=\omega$, we get $a_{0}=\omega-(4 / 81), a_{1}=-4 \omega+(10 / 27), a_{2}=6 \omega+(20 / 27)$, and $a_{3}=-4 \omega-(5 / 81)$. 
Now we propose the following 5-point ternary interpolating subdivision scheme:

$$
\begin{gathered}
f_{3 i-1}^{k+1}=a_{0} f_{i-2}^{k}+a_{1} f_{i-1}^{k}+a_{2} f_{i}^{k}+a_{3} f_{i+1}^{k}+a_{4} f_{i+2}^{k}, \\
f_{3 i}^{k+1}=f_{i}^{k}, \\
f_{3 i+1}^{k+1}=a_{4} f_{i-2}^{k}+a_{3} f_{i-1}^{k}+a_{2} f_{i}^{k}+a_{1} f_{i+1}^{k}+a_{0} f_{i+2}^{k},
\end{gathered}
$$

where $a_{0}=\omega-(4 / 81), a_{1}=-4 \omega+(10 / 27), a_{2}=6 \omega+(20 / 27)$, $a_{3}=-4 \omega-(5 / 81)$, and $a_{4}=\omega$.

3.2. Smoothness Analysis Necessary Conditions. By using the matrix formalism of Hassan and Dodgson [6], we derive necessary conditions for a scheme to be $C^{0}, C^{1}$, and $C^{2}$ by using the eigenvalues of the subdivision matrices for, midpoint rule and the vertex rule. By (14), we have the following subdivision matrices for mid-point and the vertex rules:

$$
\left(\begin{array}{cccccc}
a_{0} & a_{1} & a_{2} & a_{3} & a_{4} & 0 \\
0 & 0 & 1 & 0 & 0 & 0 \\
a_{4} & a_{3} & a_{2} & a_{1} & a_{0} & 0 \\
0 & a_{0} & a_{1} & a_{2} & a_{3} & a_{4} \\
0 & 0 & 0 & 1 & 0 & 0 \\
0 & a_{4} & a_{3} & a_{2} & a_{1} & a_{0}
\end{array}\right)
$$

(Mid-point rule)

$$
\left(\begin{array}{ccccccc}
0 & 0 & 1 & 0 & 0 & 0 & 0 \\
a_{4} & a_{3} & a_{2} & a_{1} & a_{0} & 0 & 0 \\
0 & a_{0} & a_{1} & a_{2} & a_{3} & a_{4} & 0 \\
0 & 0 & 0 & 1 & 0 & 0 & 0 \\
0 & a_{4} & a_{3} & a_{2} & a_{1} & a_{0} & 0 \\
0 & 0 & a_{0} & a_{1} & a_{2} & a_{3} & a_{4} \\
0 & 0 & 0 & 0 & 1 & 0 & 0
\end{array}\right)
$$

(Vertex rule)

where $a_{0}=\omega-(4 / 81), a_{1}=-4 \omega+(10 / 27), a_{2}=6 \omega+(20 / 27)$, $a_{3}=-4 \omega-(5 / 81)$, and $a_{4}=\omega$. The above subdivision matrices have the following eigenvalues, respectively:

$$
\begin{aligned}
& 1, \frac{1}{3}, \frac{1}{9}, \frac{1}{27}, \quad-\frac{4}{81}+11 \omega, \quad-\frac{4}{81}+3 \omega \\
& 1, \frac{1}{3}, \frac{1}{9}, \frac{1}{27}, \quad-4 \omega \\
& -6 \omega+\frac{11}{162} \pm \frac{1}{162} \sqrt{472392 \omega^{2}-9720 \omega+121}
\end{aligned}
$$

Now we find the bounds on the parameter for continuity of the scheme. From [15], the necessary condition for 5-point scheme to be $C^{0}$ is that all eigenvalues must be less than 1 . This implies that the proposed scheme is $C^{0}$ continuous for $-7 / 81<\omega<85 / 891$. The necessary condition for $C^{1}$ continuity is that all eigenvalues except 1 must be less than $1 / 3$. This is true for $(-8+2 \sqrt{10}) / 81<\omega<31 / 891$. The necessary condition for the scheme to be $C^{2}$ is that all eigenvalues except 1 and $1 / 3$ must be less than $1 / 9$. This gives the combine range $(-2+\sqrt{5}) / 81<\omega<13 / 891$ for $C^{2}$ continuity of the proposed scheme.
3.3. Smoothness Analysis Sufficient Conditions. In this section, we calculate sufficient conditions for $C^{0}, C^{1}$, and $C^{2}$ continuity of our proposed scheme by the Laurent polynomial method.

By substituting values of $a_{i}, i=0,1,2,3,4$, in $a_{i}(z)$ and then by (5), we get

$$
\begin{aligned}
& \left\|\frac{1}{3} S_{1}\right\|_{\infty}=\max \left\{\left|\omega-\frac{4}{81}\right|+\left|3 \omega-\frac{26}{81}\right|\right. \\
& +\left|3 \omega+\frac{5}{81}\right|+|\omega|,\left|\omega-\frac{4}{81}\right| \\
& +\left|3 \omega-\frac{26}{81}\right|+\left|3 \omega+\frac{5}{81}\right| \\
& \left.+|\omega|, 2|\omega|+2\left|4 \omega+\frac{1}{81}\right|+\left|6 \omega+\frac{29}{81}\right|\right\}, \\
& \left\|\frac{1}{3} S_{2}\right\|_{\infty}=\max \left\{2\left|6 \omega-\frac{4}{27}\right|+\left|12 \omega-\frac{17}{27}\right|,|9 \omega|\right. \\
& +\left|15 \omega+\frac{2}{9}\right|+\left|-\frac{1}{9}+12 \omega\right| \text {, } \\
& \left.|9 \omega|+\left|15 \omega+\frac{2}{9}\right|+\left|12 \omega-\frac{1}{9}\right|\right\} \text {, } \\
& \left\|\frac{1}{3} S_{3}\right\|_{\infty}=\max \left\{9|\omega|+\left|45 \omega-\frac{7}{9}\right|\right. \\
& +\left|36 \omega-\frac{4}{9}\right|, 2|27 \omega|+\left|54 \omega+\frac{1}{3}\right|, \\
& \left.9|\omega|+\left|45 \omega-\frac{7}{9}\right|+\left|36 \omega-\frac{4}{9}\right|\right\}, \\
& \left\|\frac{1}{3} S_{4}\right\|_{\infty}=\max \left\{|108 \omega|+\left|189 \omega-\frac{4}{3}\right|,|108 \omega|\right. \\
& \left.+\left|189 \omega-\frac{4}{3}\right|, 2|27 \omega|+\left|-216 \omega+\frac{11}{3}\right|\right\} \text {. }
\end{aligned}
$$

Since for $-7 / 81<\omega<53 / 324,\left\|(1 / 3) S_{1}\right\|_{\infty}<1$, then scheme is $C^{0}$. Similarly for $-1 / 324<\omega<2 / 81,\left\|(1 / 3) S_{2}\right\|_{\infty}<1$, then scheme is $C^{1}$, and for $1 / 324<\omega<1 / 162,\left\|(1 / 3) S_{3}\right\|_{\infty}<1$, then scheme is $C^{2}$.

$C^{3}$-continuous scheme must satisfy the condition $\left\|(1 / 3) S_{4}\right\|_{\infty}<1$; this is true for $1 / 243<\omega<7 / 891$ and $4 / 243<\omega<7 / 405$, but there is no combine range of $\omega$ for which $\left\|(1 / 3) S_{4}\right\|_{\infty}<1$. Similarly we can prove that for $L=2,3, \ldots$, we have $\left\|\left((1 / 3) S_{4}\right)^{L}\right\|_{\infty} \nless 1$; therefore the scheme is not $C^{3}$-continuous. By summarizing the above discussion, we have the following theorem.

Theorem 1. Let $\left\{f_{i}^{k}\right\}_{i \in \mathbb{Z}}$ be defined by (14) for $k \geq 0$, where $\left\{f_{i}^{0}\right\}_{i \in \mathbb{Z}}$ are given initial control points. The values correspond to $i / 3^{k}$, and let $f(t)$ be the limit function of this process, then $f(t)$ is $C^{0}, C^{1}$, and $C^{2}$-continuous for the improved ranges $-7 / 81<\omega<53 / 324,-1 / 324<\omega<2 / 81$, and $1 / 324<$ $\omega<1 / 162$, respectively. 
Remark 2. From the Laurent polynomial method, we have the improved ranges for continuity as compared to the ranges derived by using eigenanalysis method.

3.4. Basic Properties of the Scheme. The proposed 5-point scheme has the following properties.

(i) It is exact for cubic interpolation.

(ii) Its approximation order is four.

(iii) Its highest smoothness is achieved at $\omega=2 / 405$ and its Hölder exponent is $R_{H}=R(2 / 405)=2.130256$.

(iv) The basic limit function of proposed scheme has support width 7 , which implies that it vanishes outside the interval $[-7 / 2,7 / 2]$.

3.5. A Modified 5-Point Ternary Interpolating Scheme. In this section, we present a modified form of the 5-point ternary interpolating scheme which interpolates end-points without using any auxiliary control points in the case of open polygon.

Theorem 3. Let $\left\{f_{j}^{k}\right\}$ be the values corresponding to $j / 3^{k}$ for $0 \leq j \leq 3^{k} n$ at level $k \geq 0$ and $\left\{f_{j}^{0}: j=0, \ldots, n\right\}$ the set of initial control points of initial polygon $f^{0}$, then new values at level $k+1$ can be defined recursively as

$$
\begin{aligned}
f_{3 j-1}^{k+1}= & a_{0} f_{j-2}^{k}+a_{1} f_{j-1}^{k}+a_{2} f_{j}^{k} \\
& +a_{3} f_{j+1}^{k}+a_{4} f_{j+2}^{k}, \quad 2 \leq j \leq 3^{k} n-2, \\
& f_{3 j}^{k+1}=f_{j}^{k}, \quad 0 \leq j \leq 3^{k} n, \\
f_{3 j+1}^{k+1}= & a_{4} f_{j-2}^{k}+a_{3} f_{j-1}^{k}+a_{2} f_{j}^{k} \\
& +a_{1} f_{j+1}^{k}+a_{0} f_{j+2}^{k}, \quad 2 \leq j \leq 3^{k} n-2, \\
f_{1}^{k+1}= & \left(\frac{8}{21}-3 a_{0}-\frac{5}{7} a_{4}\right) f_{0}^{k} \\
& +\left(\frac{5}{3}-3 a_{1}-\frac{5}{7} a_{3}\right) f_{1}^{k}+\left(\frac{8}{3}-\frac{26}{7} a_{2}\right) f_{2}^{k} \\
& +\left(-\frac{5}{7} a_{1}-3 a_{3}\right) f_{3}^{k}+\left(-\frac{5}{7} a_{0}-3 a_{4}\right) f_{4}^{k}, \\
f_{2}^{k+1}= & \left(\frac{2}{21}-2 a_{0}-\frac{3}{7} a_{4}\right) f_{0}^{k} \\
& +\left(\frac{5}{3}-2 a_{1}-\frac{3}{7} a_{3}\right) f_{1}^{k}+\left(\frac{5}{3}-\frac{17}{7} a_{2}\right) f_{2}^{k} \\
& +\left(-\frac{3}{7} a_{1}-2 a_{3}\right) f_{3}^{k}+\left(-\frac{3}{7} a_{0}-2 a_{4}\right) f_{4}^{k},
\end{aligned}
$$

\section{Differentiability of the 5-Point Ternary Scheme}

In this section, we find exact expressions for the first and the second derivatives of limit function of the proposed scheme.

Theorem 4. For given initial control points $\left\{f_{i}^{0}\right\}$, let $\left\{f_{i}^{k}\right\}$ be the values defined by the ternary 5-point interpolating subdivision scheme (14) corresponding to $i / 3^{k} \quad(i, k \in \mathbb{Z}, k \geq$ $0)$ and $f \in C^{2}$ with $1 / 324<\omega<1 / 162$; be the corresponding limit function, then for arbitrarily fixed $m, n_{0} \in \mathbb{Z}$, the limit function $f$ has the derivatives

$$
\begin{aligned}
& f^{\prime}\left(\frac{n_{0}}{3^{m}}\right)= \frac{3^{m+k}}{144 \omega}+12 \\
& \quad \times\left\{3 \omega\left(f_{3^{k} n_{0}-3}^{m+k}-f_{3^{k} n_{0}+3}^{m+k}\right)\right. \\
& \quad+\left(f_{3^{k} n_{0}-2}^{m+k}-f_{3^{k} n_{0}+2}^{m+k}\right) \\
&\left.\quad-(81 \omega+8)\left(f_{3^{k} n_{0}-1}^{m+k}-f_{3^{k} n_{0}+1}^{m+k}\right)\right\},
\end{aligned}
$$




$$
\begin{aligned}
f^{\prime \prime}\left(\frac{n_{0}}{3^{m}}\right)= & \frac{3^{2(m+k)}}{6561 \omega^{2}+324 \omega-1} \\
\quad \times\{( & \left.729 \omega^{2}-18 \omega\right)\left(f_{3^{k} n_{0}-3}^{m+k}+f_{3^{k} n_{0}+3}^{m+k}\right) \\
& +(81 \omega-2)\left(f_{3^{k} n_{0}-2}^{m+k}+f_{3^{k} n_{0}+2}^{m+k}\right) \\
& +(162 \omega+7)\left(f_{3^{k} n_{0}-1}^{m+k}+f_{3^{k} n_{0}+1}^{m+k}\right) \\
& \left.-\left(729 \omega^{2}+225 \omega+5\right) f_{3^{k} n_{0}}^{m+k}\right\} .
\end{aligned}
$$

Proof. Let us denote

$$
F^{k}=\left(f_{3^{k} n_{0}-3}^{m+k}, f_{3^{k} n_{0}-2}^{m+k}, f_{3^{k} n_{0}-1}^{m+k}, f_{3^{k} n_{0}}^{m+k}, f_{3^{k} n_{0}+1}^{m+k}, f_{3^{k} n_{0}+2}^{m+k}, f_{3^{k} n_{0}+3}^{m+k}\right)^{T} .
$$

As the scheme is interpolatory, we have $f_{3^{k} n_{0}}^{m+k}=f_{n_{0}}^{m}$. At each subdivision step, new control points are obtained by using the rule

$$
F^{k+1}=A F^{k}
$$

where

$$
A=\left(\begin{array}{ccccccc}
0 & 0 & 1 & 0 & 0 & 0 & 0 \\
a_{4} & a_{3} & a_{2} & a_{1} & a_{0} & 0 & 0 \\
0 & a_{0} & a_{1} & a_{2} & a_{3} & a_{4} & 0 \\
0 & 0 & 0 & 1 & 0 & 0 & 0 \\
0 & a_{4} & a_{3} & a_{2} & a_{1} & a_{0} & 0 \\
0 & 0 & a_{0} & a_{1} & a_{2} & a_{3} & a_{4} \\
0 & 0 & 0 & 0 & 1 & 0 & 0
\end{array}\right),
$$

is vertex subdivision matrix with $a_{0}=\omega-(4 / 81), a_{1}=$ $-4 \omega+(10 / 27)$, and $a_{2}=6 \omega+(20 / 27), a_{3}=-4 \omega-$ (5/81), $a_{4}=\omega$. Matrix $A$ has seven eigenvalues $\lambda_{1}=1$, $\lambda_{2}=1 / 3, \lambda_{3}=1 / 9, \lambda_{4}=1 / 27, \lambda_{5}=-6 \omega+(11 / 162)+$ $(1 / 162) \sqrt{472392 \omega^{2}-9720 \omega+121}, \lambda_{6}=-4 \omega$, and $\lambda_{7}=$ $-6 \omega+(11 / 162)-1 / 162 \sqrt{472392 \omega^{2}-9720 \omega+121}$ and has seven orthogonal eigenvectors. Let $\hat{r}_{i}$ and $\hat{l}_{i}$ be the right and left eigenvectors of matrix $A$ corresponding to the eigenvalues $\lambda_{i}, i=1,2,3, \ldots, 7$, then direct computation leads to

$$
\begin{aligned}
& \hat{r}_{1}=\left(\begin{array}{l}
1 \\
1 \\
1 \\
1 \\
1 \\
1 \\
1
\end{array}\right), \quad \hat{r}_{2}=\left(\begin{array}{c}
-3 \\
-2 \\
-1 \\
0 \\
1 \\
2 \\
3
\end{array}\right), \quad \hat{r}_{3}=\left(\begin{array}{l}
9 \\
4 \\
1 \\
0 \\
1 \\
4 \\
9
\end{array}\right), \quad \hat{r}_{4}=\left(\begin{array}{c}
-27 \\
-8 \\
-1 \\
0 \\
1 \\
8 \\
27
\end{array}\right) \text {, } \\
& \hat{r}_{5}=\left(\begin{array}{c}
1 \\
\frac{-729 \omega^{2}-60 \omega+162 \lambda_{5} \omega+(77 / 162)-(7 / 162) \sqrt{472392 \omega^{2}-9720 \omega+121}}{81 \omega-2} \\
\lambda_{5} \\
0 \\
\lambda_{5} \\
\frac{-729 \omega^{2}-60 \omega+162 \lambda_{5} \omega+(77 / 162)-(7 / 162) \sqrt{472392 \omega^{2}-9720 \omega+121}}{81 \omega-2} \\
1
\end{array}\right), \quad \hat{r}_{6}=\left(\begin{array}{r}
-1 \\
324 \omega^{2}+35 \omega \\
4 \omega \\
0 \\
-4 \omega \\
-\left(324 \omega^{2}+35 \omega\right) \\
1
\end{array}\right), \\
& \hat{r}_{7}=\left(\begin{array}{c}
1 \\
\frac{-729 \omega^{2}-60 \omega+162 \lambda_{7} \omega+(77 / 162)+(7 / 162) \sqrt{472392 \omega^{2}-9720 \omega+121}}{81 \omega-2} \\
\lambda_{7} \\
0 \\
\lambda_{7} \\
\frac{-729 \omega^{2}-60 \omega+162 \lambda_{7} \omega+(77 / 162)+(7 / 162) \sqrt{472392 \omega^{2}-9720 \omega+121}}{81 \omega-2} \\
1
\end{array}\right),
\end{aligned}
$$


and left eigenvectors are

$$
\begin{aligned}
\hat{l}_{2}= & \left(\frac{3 \omega}{144 \omega+12}, \frac{1}{144 \omega+12}, \frac{-(81 \omega+8)}{144 \omega+12},\right. \\
& \left.0, \frac{81 \omega+8}{144 \omega+12}, \frac{-1}{144 \omega+12}, \frac{-3 \omega}{144 \omega+12}\right), \\
\hat{l}_{3}= & \left(\frac{9 \omega(81 \omega-2)}{2\left(6561 \omega^{2}+324 \omega-1\right)}, \frac{81 \omega-2}{2\left(6561 \omega^{2}+324 \omega-1\right)},\right. \\
& \frac{162 \omega+7}{2\left(6561 \omega^{2}+324 \omega-1\right)},
\end{aligned}
$$

$$
\begin{aligned}
& \frac{-\left(729 \omega^{2}+225 \omega+5\right)}{6561 \omega^{2}+324 \omega-1}, \frac{162 \omega+7}{2\left(6561 \omega^{2}+324 \omega-1\right)}, \\
& \left.\frac{81 \omega-2}{2\left(6561 \omega^{2}+324 \omega-1\right)}, \frac{9 \omega(81 \omega-2)}{2\left(6561 \omega^{2}+324 \omega-1\right)}\right) .
\end{aligned}
$$

If the limit curve generated by 5-point ternary interpolating subdivision scheme defines a $C^{2}$-continuous function for $1 / 324<\omega<1 / 162$, then necessarily

$$
\begin{gathered}
\lim _{k \rightarrow \infty} F^{k}=f_{n_{0}}^{m} \widehat{r}_{1}=f\left(\frac{n_{0}}{3^{m}}\right) \widehat{r}_{1}, \\
\lim _{k \rightarrow \infty} \frac{f_{3^{k} n_{0}+j}^{m+k}-f_{n_{0}}^{m}}{j / 3^{m+k}} \\
=\lim _{k \rightarrow \infty} \frac{f\left(\left(3^{k} n_{0}+j\right) / 3^{m+k}\right)-f\left(n_{0} / 3^{m}\right)}{j / 3^{m+k}} \\
=f^{\prime}\left(\frac{n_{0}}{3^{m}}\right), \quad j= \pm 1, \pm 2, \pm 3, \\
\lim _{k \rightarrow \infty} \frac{f_{3^{k} n_{0}+j}^{m+k}+f_{3^{k} n_{0}-j}^{m+k}-2 f_{n_{0}}^{m}}{\left(j / 3^{m+k}\right)^{2}} \\
=\lim _{k \rightarrow \infty} \frac{f\left(\left(3^{k} n_{0}+j\right) / 3^{m+k}\right)+f\left(\left(3^{k} n_{0}-j\right) / 3^{m+k}\right)-2 f\left(n_{0} / 3^{m}\right)}{\left(j / 3^{m+k}\right)^{2}} \\
=f^{\prime \prime}\left(\frac{n_{0}}{3^{m}}\right), \quad j= \pm 1, \pm 2, \pm 3 .
\end{gathered}
$$

So we have

$$
\lim _{k \rightarrow \infty} 3^{m+k} D\left(F^{k}-f_{n_{0}}^{m} \widehat{r}_{1}\right)=f^{\prime}\left(\frac{n_{0}}{3^{m}}\right) \hat{e}
$$

where $\hat{e}=(1,1,1,0,1,1,1)^{T}, D=\operatorname{diag}(-1 / 3,-1 / 2,-1,1,1$, $1 / 2,1 / 3)$. This implies that

$$
\lim _{k \rightarrow \infty} 3^{2(m+k)} D^{2}\left(F^{k}+J F^{k}-2 f_{n_{0}}^{m} \hat{r}_{1}\right)=f^{\prime \prime}\left(\frac{n_{0}}{3^{m}}\right) \hat{e}
$$

where $D^{2}=\operatorname{diag}(1 / 9,1 / 4,1,1,1,1 / 4,1 / 9)$. Since matrix $A$ has linearly independent eigenvectors, there exist scalars $\alpha_{1}, \alpha_{2}, \ldots, \alpha_{7}$, such that $F^{0}$ can be written as

$$
F^{0}=\sum_{i=1}^{7} \alpha_{i} \widehat{r}_{i}
$$

This implies that $\alpha_{1}=f_{n_{0}}^{m}$; therefore $F^{k}$ can be written as

$$
\begin{aligned}
F^{k}= & A^{k} F^{0}=\lambda^{k} F^{0}=\sum_{i=1}^{7} \lambda_{i}^{k} \alpha_{i} \hat{r}_{i} \\
= & f_{n_{0}}^{m} \hat{r}_{1}+\alpha_{2}\left(\frac{1}{3}\right)^{k} \hat{r}_{2}+\alpha_{3}\left(\frac{1}{9}\right)^{k} \hat{r}_{3}+\alpha_{4}\left(\frac{1}{27}\right)^{k} \hat{r}_{4} \\
& +\sum_{i=5}^{7} \alpha_{i}\left(\lambda_{i}\right)^{k} \hat{r}_{i} .
\end{aligned}
$$

By using (43) in (40), we have

$$
\begin{aligned}
f^{\prime}\left(\frac{n_{0}}{3^{m}}\right) \hat{e}=\lim _{k \rightarrow \infty} 3^{m+k} D\left(F^{k}-f_{n_{0}}^{m} \hat{r}_{1}\right) \\
=\lim _{k \rightarrow \infty} 3^{m+k} D\left\{f_{n_{0}}^{m} \widehat{r}_{1}+\alpha_{2}\left(\frac{1}{3}\right)^{k} \widehat{r}_{2}\right. \\
+\alpha_{3}\left(\frac{1}{9}\right)^{k} \hat{r}_{3}+\alpha_{4}\left(\frac{1}{27}\right)^{k} \hat{r}_{4} \\
\left.+\sum_{i=5}^{7} \alpha_{i}\left(\lambda_{i}\right)^{k} \widehat{r}_{i}-f_{n_{0}}^{m} \widehat{r}_{1}\right\}
\end{aligned}
$$




$$
\begin{gathered}
=\lim _{k \rightarrow \infty} 3^{m}\left\{\alpha_{2} D \widehat{r}_{2}+\alpha_{3}\left(\frac{1}{3}\right)^{k} D \widehat{r}_{3}+\alpha_{4}\left(\frac{1}{9}\right)^{k} D \widehat{r}_{4}\right. \\
\left.+3^{k} \sum_{i=5}^{7} \alpha_{i}\left(\lambda_{i}\right)^{k} D \widehat{r}_{i}\right\} .
\end{gathered}
$$

This implies that

$$
f^{\prime}\left(\frac{n_{0}}{3^{m}}\right) \hat{e}=3^{m} \alpha_{2} D \widehat{r}_{2}
$$

In view of

$$
J \hat{r}_{i}= \begin{cases}\hat{r}_{i}, & i=1,3,5,7, \\ -\hat{r}_{i}, & i=2,4,6,\end{cases}
$$

and using (43) in (41) we have

$$
\begin{aligned}
f^{\prime \prime}\left(\frac{n_{0}}{3^{m}}\right) \hat{e}= & \lim _{k \rightarrow \infty} 3^{2(m+k)} D^{2}\left(F^{k}+J F^{k}-2 f_{n_{0}}^{m} \hat{r}_{1}\right) \\
= & \lim _{k \rightarrow \infty} 3^{2(m+k)} D^{2} \\
& \times\left\{f_{n_{0}}^{m} \hat{r}_{1}+\alpha_{2}\left(\frac{1}{3}\right)^{k} \hat{r}_{2}+\alpha_{3}\left(\frac{1}{9}\right)^{k} \hat{r}_{3}\right. \\
& +\alpha_{4}\left(\frac{1}{27}\right)^{k} \hat{r}_{4}+\sum_{i=5}^{7} \alpha_{i}\left(\lambda_{i}\right)^{k} \hat{r}_{i}+f_{n_{0}}^{m} \hat{r}_{1} \\
& -\alpha_{2}\left(\frac{1}{3}\right)^{k} \hat{r}_{2}+\alpha_{3}\left(\frac{1}{9}\right)^{k} \hat{r}_{3} \\
& -\alpha_{4}\left(\frac{1}{27}\right)^{k} \hat{r}_{4}+\alpha_{5}\left(\lambda_{5}\right)^{k} \hat{r}_{5} \\
& \left.-\alpha_{6}\left(\lambda_{6}\right)^{k} \hat{r}_{6}+\alpha_{7}\left(\lambda_{7}\right)^{k} \hat{r}_{7}-2 f_{n_{0}}^{m} \hat{r}_{1}\right\} .
\end{aligned}
$$

By simplification, we get

$$
\begin{aligned}
& f^{\prime \prime}\left(\frac{n_{0}}{3^{m}}\right) \hat{e} \\
& =\lim _{k \rightarrow \infty} 3^{2 m}\left(2 \alpha_{3} D^{2} \widehat{r}_{3}+2 \alpha_{5}\left(9 \lambda_{5}\right)^{k} D^{2} \hat{r}_{5}+2 \alpha_{7}\left(9 \lambda_{7}\right)^{k} D^{2} \widehat{r}_{7}\right) .
\end{aligned}
$$

So, we have

$$
f^{\prime \prime}\left(\frac{n_{0}}{3^{m}}\right) \hat{e}=3^{2 m} 2 \alpha_{3} D^{2} \widehat{r}_{3}
$$

Since we have

$$
D \widehat{r}_{2}=\left(\begin{array}{ccccccc}
-\frac{1}{3} & 0 & 0 & 0 & 0 & 0 & 0 \\
0 & -\frac{1}{2} & 0 & 0 & 0 & 0 & 0 \\
0 & 0 & -1 & 0 & 0 & 0 & 0 \\
0 & 0 & 0 & 1 & 0 & 0 & 0 \\
0 & 0 & 0 & 0 & 1 & 0 & 0 \\
0 & 0 & 0 & 0 & 0 & \frac{1}{2} & 0 \\
0 & 0 & 0 & 0 & 0 & 0 & \frac{1}{3}
\end{array}\right)\left(\begin{array}{c}
-3 \\
-2 \\
-1 \\
0 \\
1 \\
2 \\
3
\end{array}\right)=\left(\begin{array}{l}
1 \\
1 \\
1 \\
0 \\
1 \\
1 \\
1
\end{array}\right)=\widehat{e}
$$

$$
D^{2} \widehat{r}_{3}=\left(\begin{array}{ccccccc}
\frac{1}{9} & 0 & 0 & 0 & 0 & 0 & 0 \\
0 & \frac{1}{4} & 0 & 0 & 0 & 0 & 0 \\
0 & 0 & 1 & 0 & 0 & 0 & 0 \\
0 & 0 & 0 & 1 & 0 & 0 & 0 \\
0 & 0 & 0 & 0 & 1 & 0 & 0 \\
0 & 0 & 0 & 0 & 0 & \frac{1}{4} & 0 \\
0 & 0 & 0 & 0 & 0 & 0 & \frac{1}{9}
\end{array}\right)\left(\begin{array}{l}
9 \\
4 \\
1 \\
0 \\
1 \\
4 \\
9
\end{array}\right)=\left(\begin{array}{l}
1 \\
1 \\
1 \\
0 \\
1 \\
1 \\
1
\end{array}\right)=\hat{e}
$$

By using the above facts in (45) and (49), we get

$$
\begin{gathered}
f^{\prime}\left(\frac{n_{0}}{3^{m}}\right)=3^{m} \alpha_{2}, \\
f^{\prime \prime}\left(\frac{n_{0}}{3^{m}}\right)=2(3)^{2 m} \alpha_{3} .
\end{gathered}
$$

Now by multiplying (43) with the left eigenvector $\hat{l}_{2}$, corresponding to eigenvalue $\lambda_{2}=1 / 3$, and in view of

$$
\widehat{l}_{j} \hat{r}_{i}= \begin{cases}1, & i=j, \\ 0, & i \neq j,\end{cases}
$$

we have

$$
\begin{array}{r}
\hat{l}_{2} F^{k}=\hat{l}_{2}\left(f_{n_{0}}^{m} \hat{r}_{1}+\alpha_{2}\left(\frac{1}{3}\right)^{k} \hat{r}_{2}+\alpha_{3}\left(\frac{1}{9}\right)^{k} \hat{r}_{3}\right. \\
\left.+\alpha_{4}\left(\frac{1}{27}\right)^{k} \hat{r}_{4}+\sum_{i=5}^{7} \alpha_{i}\left(\lambda_{i}\right)^{k} \hat{r}_{i}\right) .
\end{array}
$$

This implies that

$$
\hat{l}_{2} F^{k}=\alpha_{2}\left(\frac{1}{3}\right)^{k}
$$

So, we have

$$
\alpha_{2}=3^{k} \hat{l}_{2} F^{k}
$$

Using the above result in (52), we have

$$
f^{\prime}\left(\frac{n_{0}}{3^{m}}\right)=3^{m} \alpha_{2}=3^{m} 3^{k} \widehat{r}_{2} F^{k} .
$$


Now by (29) and using the value of $\hat{l}_{2}$, we have

$$
f^{\prime}\left(\frac{n_{0}}{3^{m}}\right)=\frac{3^{m+k}}{144 w+12}\left(\begin{array}{c}
3 \omega \\
1 \\
-(81 \omega+8) \\
0 \\
81 \omega+8) \\
-1 \\
-3 \omega
\end{array}\right)\left(\begin{array}{c}
f_{3^{k} n_{0}-3}^{m+k} \\
f_{3^{k} n_{0}-2}^{m+k} \\
f_{3^{k} n_{0}-1}^{m+k} \\
f_{3^{k} n_{0}}^{m+k} \\
f_{3^{k} n_{0}+1}^{m+k} \\
f_{3^{k} n_{0}+2}^{m+k} \\
f_{3^{k} n_{0}+3}^{m+k}
\end{array}\right) .
$$

By simplification, we get

$$
\begin{aligned}
& f^{\prime}\left(\frac{n_{0}}{3^{m}}\right)= \frac{3^{m+k}}{144 \omega}+12 \\
& \quad \times\left\{3 \omega\left(f_{3^{k} n_{0}-3}^{m+k}-f_{3^{k} n_{0}+3}^{m+k}\right)\right. \\
& \quad+\left(f_{3^{k} n_{0}-2}^{m+k}-f_{3^{k} n_{0}+2}^{m+k}\right)-(81 \omega+8) \\
&\left.\quad \times\left(f_{3^{k} n_{0}-1}^{m+k}-f_{3^{k} n_{0}+1}^{m+k}\right)\right\} .
\end{aligned}
$$

Similarly, by multiplying (43) with the left eigenvector $\hat{l}_{3}$, corresponding to $\lambda_{3}=1 / 9$, we get

$$
\hat{l}_{3} F^{k}=\alpha_{3}\left(\frac{1}{9}\right)^{k} \text {. }
$$

This implies that

$$
\alpha_{3}=9^{k} \hat{l}_{3} F^{k}=3^{2 k} \hat{l}_{3} F^{k}
$$

Using the above equation in (53), we have

$$
f^{\prime \prime}\left(\frac{n_{0}}{3^{m}}\right)=3^{2(m+k)} 2 \hat{l}_{3} F^{k}
$$

So by (29) and using the value of, $\hat{l}_{3}$, we have

$$
f^{\prime \prime}\left(\frac{n_{0}}{3^{m}}\right)=\frac{3^{2(m+k)}}{6561 \omega^{2}+324 \omega-1}
$$

$$
\times\left(\begin{array}{c}
9 \omega(81 \omega-2) \\
81 \omega-2 \\
162 \omega+7 \\
-729 \omega^{2}-225 \omega-5 \\
162 \omega+7 \\
81 \omega-2 \\
9 \omega(81 \omega-2)
\end{array}\right)\left(\begin{array}{c}
f_{3^{k} n_{0}-3}^{m+k} \\
f_{3^{k} n_{0}-2}^{m+k} \\
f_{3^{k} n_{0}-1}^{m+k} \\
f_{3^{k} n_{0}}^{m+k} \\
f_{3^{k} n_{0}+1}^{m+k} \\
f_{3^{k} n_{0}+2}^{m+k} \\
f_{3^{k} n_{0}+3}^{m+k}
\end{array}\right)
$$

TABle 1: Comparison of proposed 5-point ternary scheme with some of existing binary and ternary schemes.

\begin{tabular}{lcccc}
\hline Subdivision scheme & Type & $\begin{array}{c}\text { Approximation } \\
\text { order }\end{array}$ & Support & $C^{n}$ \\
\hline 4-point binary [10] & Interpolating & 4 & 6 & 1 \\
4-point binary [2] & Interpolating & 4 & 6 & 1 \\
DD 4-point binary & Interpolating & 3 & 3 & 1 \\
[3] & Interpolating & 3 & 5 & 2 \\
4-point ternary [9] & Interpolating & 3 & 5 & 2 \\
4-point ternary [5] & Interpolating & 6 & 7 & 1 \\
5-point ternary [13] & Interpolating & 4 & 7 & 2 \\
$\begin{array}{l}\text { Proposed 5-point } \\
\text { ternary }\end{array}$ & Interpolating & 4 & & \\
\hline
\end{tabular}

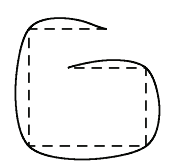

(a)

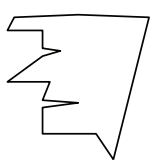

(b)

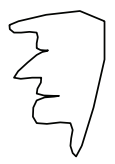

(c)

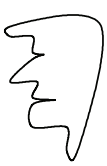

(d)
Figure 1: (a) The dashed lines show initial polygon while the solid curve is constructed by the proposed scheme with parameter $\omega=$ $1 / 200$, (b) initial polygon, (c) result after first subdivision step, and (d) result after 2 nd subdivision step.

After simplification, we get

$$
\begin{aligned}
f^{\prime \prime}\left(\frac{n_{0}}{3^{m}}\right)= & \frac{3^{2(m+k)}}{6561 \omega^{2}+324 \omega-1} \\
\quad \times\{( & \left.729 \omega^{2}-18 \omega\right) \\
& \times\left(f_{3^{k} n_{0}-3}^{m+k}+f_{3^{k} n_{0}+3}^{m+k}\right) \\
& +(81 \omega-2)\left(f_{3^{k} n_{0}-2}^{m+k}+f_{3^{k} n_{0}+2}^{m+k}\right) \\
& +(162 \omega+7)\left(f_{3^{k} n_{0}-1}^{m+k}+f_{3^{k} n_{0}+1}^{m+k}\right) \\
& \left.-\left(729 \omega^{2}+225 \omega+5\right) f_{3^{k} n_{0}}^{m+k}\right\} .
\end{aligned}
$$

\section{Comparison and Application of the Scheme}

We give the comparison of some properties of the proposed 5 -point ternary scheme with other existing binary and ternary schemes in Table 1.

In this section, we also display the performance of the proposed schemes (14) and the scheme introduced in Theorem 3, by applying these schemes on open and closed polygons. Figure 1(a) shows initial open polygon and its limit curve. In this figure we also show the initial closed polygon and the results after first and 2 nd subdivision level with parametric value $\omega=1 / 200$. 


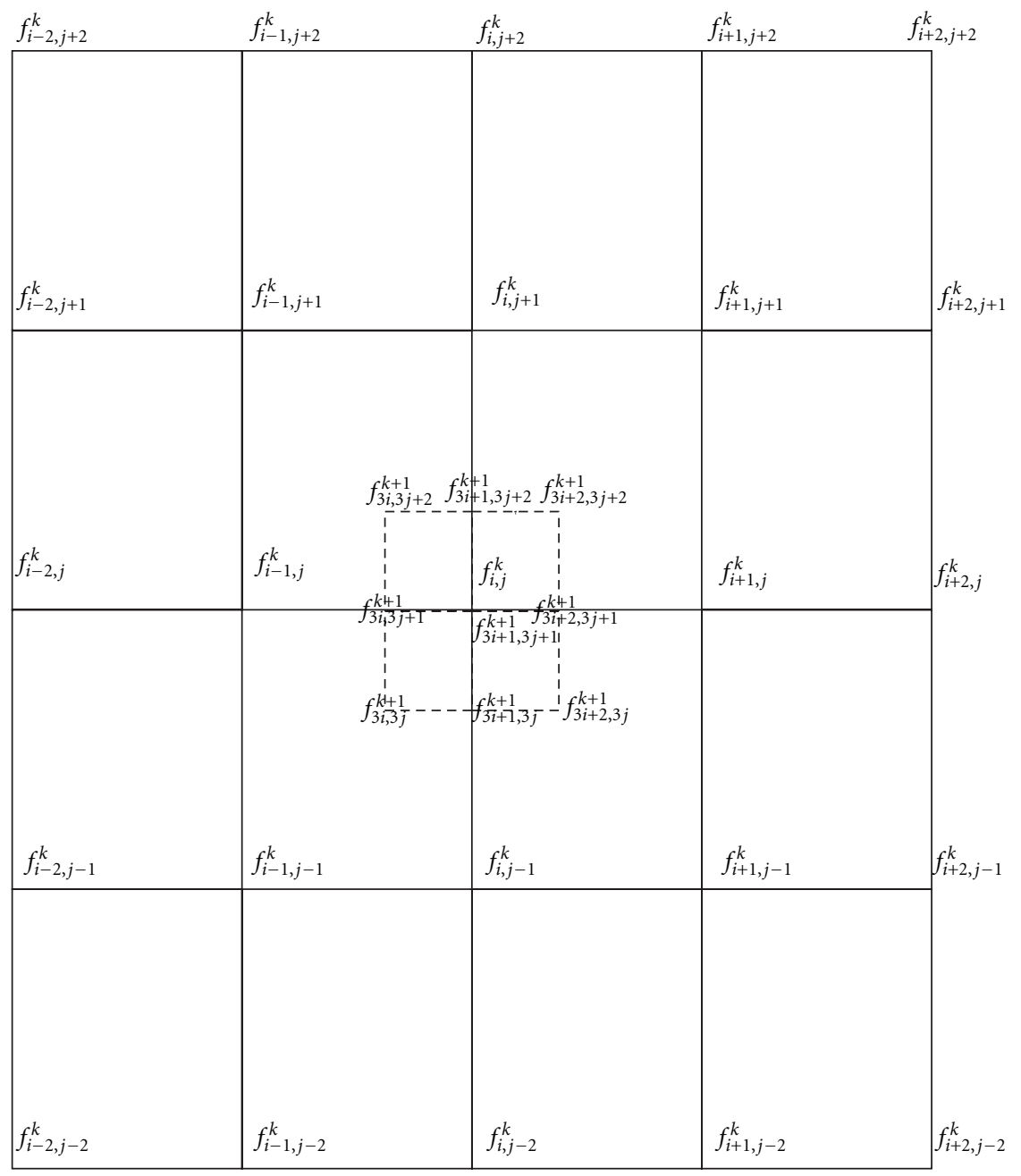

FIGURE 2: Solid lines show initial polygon while dotted lines are refined polygon.

\section{A 5-Point Tensor Product Ternary Scheme}

In this section, we present a 5-point tensor product ternary interpolating subdivision scheme. Some numerical examples of this tensor product scheme are also included. By taking the tensor product of scheme (14), we have the following nine rules representing a 5-point tensor product ternary interpolating scheme:

$$
\begin{gathered}
f_{3 i, 3 j}^{k+1}=\sum_{s=0}^{4} \sum_{t=0}^{4} a_{s} a_{t} f_{i+s-2, j+t-2}^{k}, \\
f_{3 i, 3 j+1}^{k+1}=\sum_{s=0}^{4} a_{s} f_{i+s-2, j}^{k}, \\
f_{3 i, 3 j+2}^{k+1}=\sum_{s=0}^{4} \sum_{t=0}^{4} a_{s} a_{4-t} f_{i+s-2, j+t-2}^{k}, \\
f_{3 i+1,3 j}^{k+1}=\sum_{s=0}^{4} a_{s} f_{i, j+s-2}^{k},
\end{gathered}
$$

$$
\begin{gathered}
f_{3 i+1,3 j+1}^{k+1}=f_{i, j}^{k}, \\
f_{3 i+1,3 j+2}^{k+1}=\sum_{s=0}^{4} a_{4-s} f_{i, j+s-2}^{k}, \\
f_{3 i+2,3 j}^{k+1}=\sum_{s=0}^{4} \sum_{t=0}^{4} a_{4-s} a_{t} f_{i+s-2, j+t-2}^{k}, \\
f_{3 i+2,3 j+1}^{k+1}=\sum_{s=0}^{4} a_{4-s} f_{i+s-2, j}^{k}, \\
f_{3 i+2,3 j+2}^{k+1}=\sum_{s=0}^{4} \sum_{t=0}^{4} a_{4-s} a_{4-t} f_{i+s-2, j+t-2}^{k} .
\end{gathered}
$$

Figure 2 shows how does the proposed 5-point tensor product ternary interpolating scheme work on control polygon with control points $f_{i, j}^{k}, i, j \in \mathbb{R}^{N}, N \geq 2$ ? In this figure, solid lines show the faces of initial polygon while dotted lines show the faces of refined polygon. 


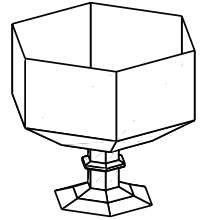

(a)

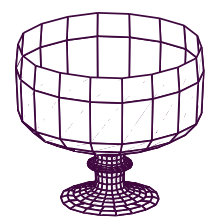

(b)

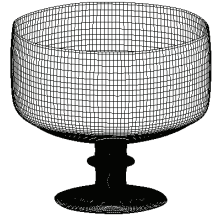

(c)
Figure 3: (a) Initial polygon, (b) result after first level, (c) result after the second level.

Figure 3(a) shows the outline of the cup like shape which is considered to be an initial control polygon of proposed scheme. Figures 3(b) and 3(c) show the results of the proposed scheme after first and second subdivision levels. This figure shows that the results of a 5-point tensor product ternary interpolating scheme are visually smooth.

\section{Conclusion}

In this paper, we have presented a new 5-point ternary interpolating subdivision scheme with one parameter. The differentiability of the proposed scheme is also calculated. Some important properties of the scheme such as support, approximation order, conditions of $C^{0}-, C^{1}-, C^{2}$-continuity, and Hölders exponent are derived. A modified 5-point ternary scheme to deal with open polygons is also proposed to enhance the modeling ability of the scheme. Comparison of the proposed scheme with the other existing scheme is given. Some numerical examples are presented to show the visual performance of our scheme. In this paper, we have also introduced a 5-point tensor product interpolating scheme.

\section{Acknowledgment}

This work is supported by the Indigenous Ph.D. Scholarship Scheme of the Higher Education Commission (HEC), Pakistan.

\section{References}

[1] S. Dubuc, "Interpolation through an iterative scheme," Journal of Mathematical Analysis and Applications, vol. 114, no. 1, pp. 185-204, 1986.

[2] N. Dyn, D. Levin, and J. A. Gregory, "A 4-point interpolatory subdivision scheme for curve design," Computer Aided Geometric Design, vol. 4, no. 4, pp. 257-268, 1987.

[3] G. Deslauriers and S. Dubuc, "Symmetric iterative interpolation processes," Constructive Approximation, vol. 5, no. 1, pp. 49-68, 1989.

[4] A. Weissman, A 6-point interpolatory subdivision scheme for curve design [M.S. thesis], Tel-Aviv University, Tel Aviv, Israel, 1990.

[5] M. F. Hassan, I. P. Ivrissimitzis, N. A. Dodgson, and M. A. Sabin, "An interpolating 4-point $C^{2}$ ternary stationary subdivision scheme," Computer Aided Geometric Design, vol. 19, no. 1, pp. 1-18, 2002.
[6] M. F. Hassan and N. A. Dodgson, "Ternary three point univariate subdivision scheme," in Curve and Surface Fitting: Saint-Malo, A. Cohen, J. Laouis Merrien, and L. L. Schumaker, Eds., pp. 199-208, 2002.

[7] M. F. Hassan and N. A. Dodgson, "Further analysis of ternary three point univariate subdivision scheme," Tech. Rep. 599, University of Cambridge Computer Laboratory, 2004.

[8] N. Dyn, Tutorials on Multiresolution in Geometric Modelling, Summer School Lecture Notes Series: Mathematics and Visualization, Springer, Berlin, Germany, 2002.

[9] C. Beccari, G. Casciola, and L. Romani, "An interpolating 4point $C^{2}$ ternary non-stationary subdivision scheme with tension control," Computer Aided Geometric Design, vol. 24, no. 4, pp. 210-219, 2007.

[10] C. Beccari, G. Casciola, and L. Romani, "A non-stationary uniform tension controlled interpolating 4-point scheme reproducing conics," Computer Aided Geometric Design, vol. 24, no. 1, pp. 1-9, 2007.

[11] K. P. Ko, A Study on Subdivision Scheme, Dongseo University, Busan, Republic of Korea, 2007.

[12] H. Zheng, H. Zhao, Z. Ye, and M. Zhou, "Differentiability of four point ternary subdivision scheme and its application, LEANG," International Journal of Computer Science, vol. 36, no. 1, pp. 1-4, 2007.

[13] J.-A. Lian, "On a-ary subdivision for curve design: II. 3-point and 5-point interpolatory schemes," Application and Applied Mathematics, vol. 3, no. 1, pp. 176-187, 2008.

[14] C. Conti, L. Gemignani, and L. Romani, "From symmetric subdivision masks of hurwitz type to interpolatory subdivision masks," Linear Algebra and Its Applications, vol. 431, no. 10, pp. 1971-1987, 2009.

[15] M. A. Sabin, "Eigenanalysis and artifacts of subdivision curves and surfaces," in Tutorials on Multiresolution in Geometric Modelling, A. Iske, E. Quak, and M. S. Floater, Eds., chapter 4, pp. 69-92, Springer, Berlin, Germany, 2002. 


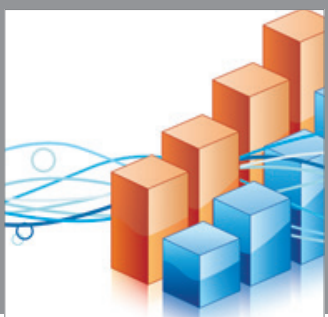

Advances in

Operations Research

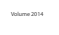

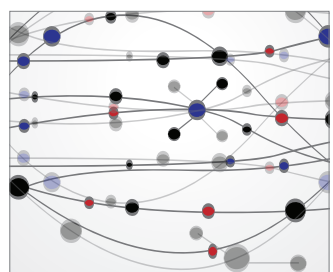

\section{The Scientific} World Journal
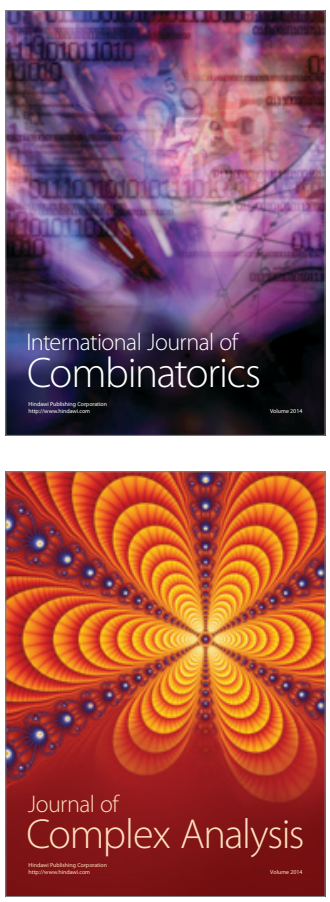

International Journal of

Mathematics and

Mathematical

Sciences
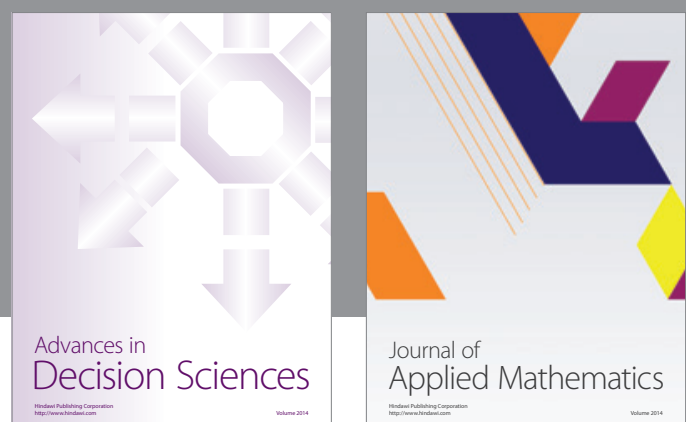

Journal of

Applied Mathematics
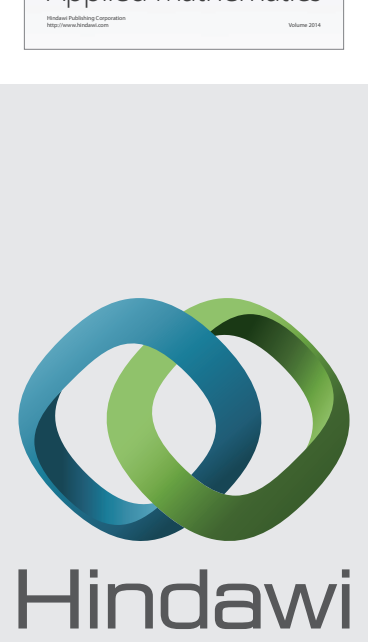

Submit your manuscripts at http://www.hindawi.com
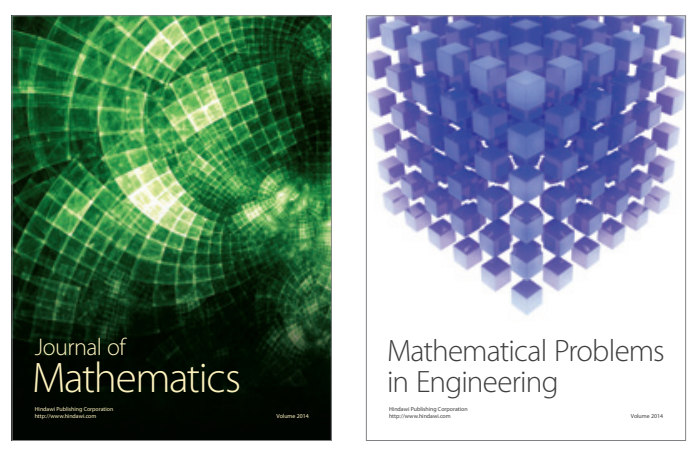

Mathematical Problems in Engineering
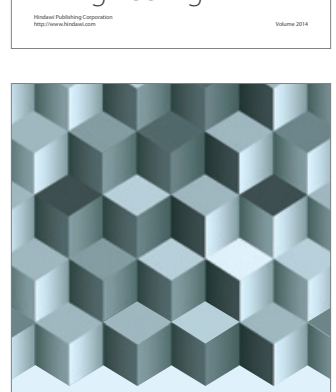

Journal of

Function Spaces
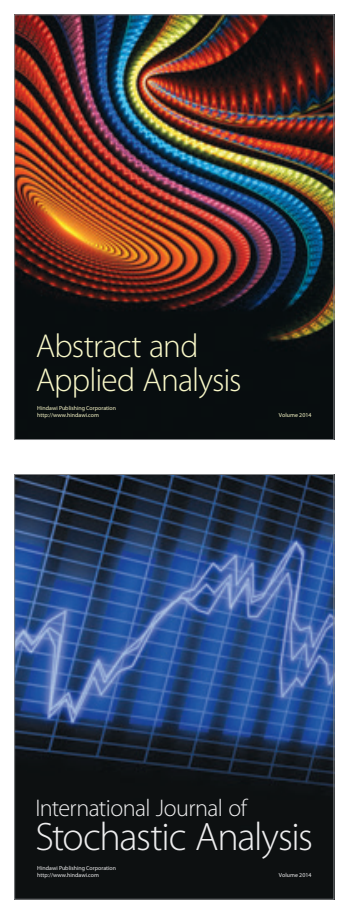

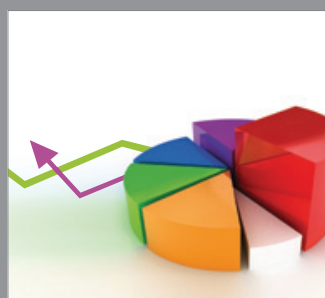

ournal of

Probability and Statistics

Promensencen
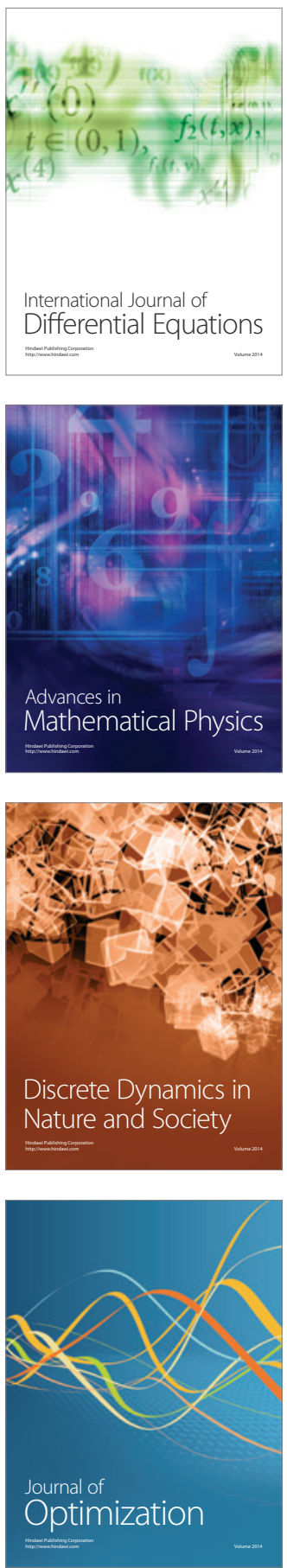